\title{
Not the usual suspect
}

Filipa Cardoso ${ }^{1}$, Rui Barata ${ }^{1}$, Joaquim Calado ${ }^{1}$, Mário Góis ${ }^{1,2}$, Helena Viana ${ }^{1,2}$, David Navarro ${ }^{1}$, Marco Mendes ${ }^{1}$, Fernando Nolasco $^{1}$

${ }^{1}$ Nephrology Department, Hospital de Curry Cabral - Centro Hospitalar de Lisboa Central

2 Laboratory of Renal Morphology, Nephrology Department, Hospital Curry Cabral - Centro Hospitalar de Lisboa Central

\section{CLINICAL PRESENTATION}

We present a case of a 69-year-old caucasian male with prior type 2 diabetes mellitus and immune thrombocytopenia treated with corticosteroids and splenectomy in 2015. The patient had been hospitalized for one month prior to our evaluation with fever, anorexia, fatigue and abdominal pain which had started 2 weeks before. The initial laboratory tests showed anemia (hemoglobin $8.7 \mathrm{~g} / \mathrm{L}$ ) and elevated inflammatory parameters [leukocytes $35 \times 10^{\wedge} 9 / \mathrm{L}$, neutrophilia $29 \times 10^{\wedge} 9 / \mathrm{L}$ and $\mathrm{c}$-reactive protein $\left.285 \mathrm{mg} / \mathrm{L}\right]$, for which he performed a CT-scan that revealed an abdominal abscess of unknown origin. He was treated empirically with meropenem for two weeks with clinical improvement, although his inflammatory parameters remained elevated. Blood cultures were negative. Simultaneously, his previous normal kidney function [creatinine $(\mathrm{Scr}) 0.9 \mathrm{mg} / \mathrm{dL}$ ] started to deteriorate rapidly (Scr $5.63 \mathrm{mg} / \mathrm{dL}$ at our observation). Urinalysis showed hematuria (3569/uL) and urine sediment was suggestive of a glomerular origin. Kidney ultrasound was unremarkable. The workup for possible glomerulonephritis (GN) revealed a positive ANCA-PR3 title of $9092 \mathrm{UQ}$ and a protein/creatinine ratio of $0.6 \mathrm{~g} / \mathrm{g}$. Given that his inflammatory parameters remained elevated even after a course of broad-spectrum antibiotics and that blood cultures were consistently negative, it was highly unlikely this was due to an infection. With a strong suspicion of pauci-immune vasculitis, he was started on intravenous methylprednisolone $(1 \mathrm{~g} /$ day) for three consecutive days, followed by oral prednisolone $(60 \mathrm{mg} /$ day) and intravenous cyclophosphamide (750mg), and scheduled for a renal biopsy as soon as possible. This treatment led to a steep decrease of the inflammatory parameters but his Scr deteriorated further and dialysis was needed. After two doses of cyclophosphamide, and given the dependence on dialysis, we decided to initiate plasmapheresis as a last resort.

\section{Figure 2}

Periodic acid-Schiff $\times 400$

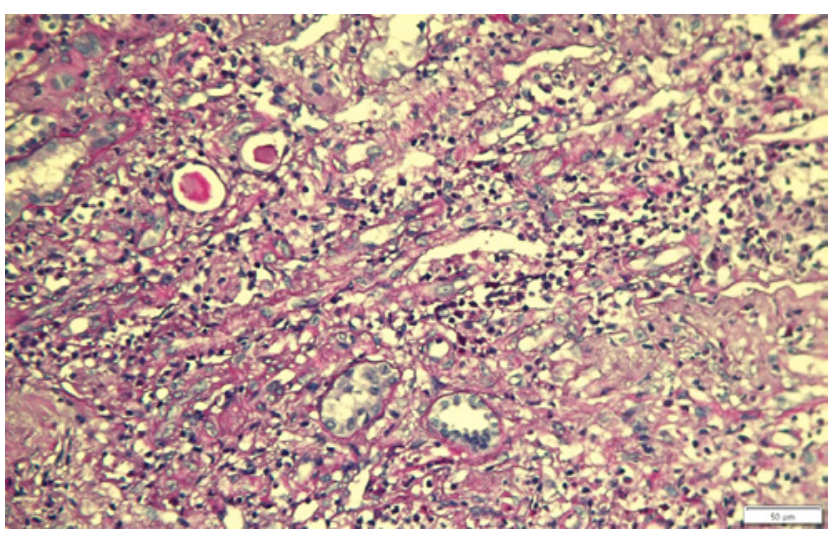

Figure 3

Masson's trichrome $x 400$

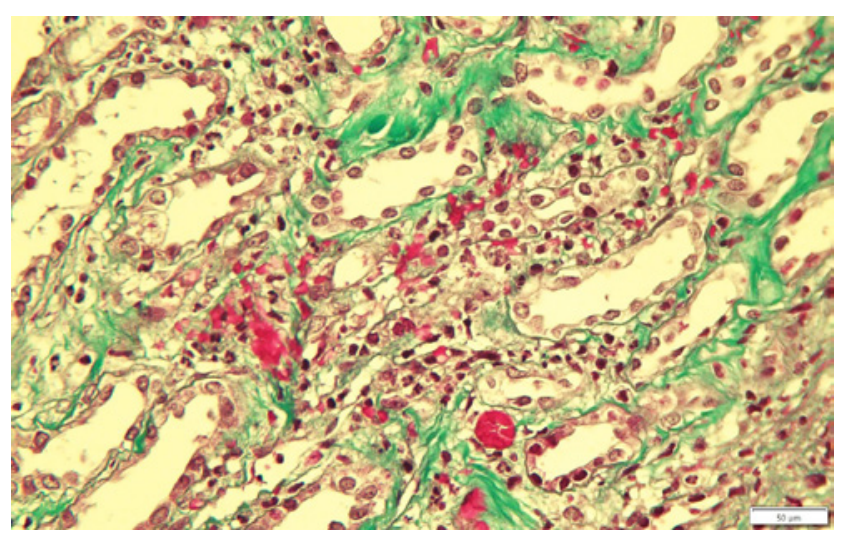




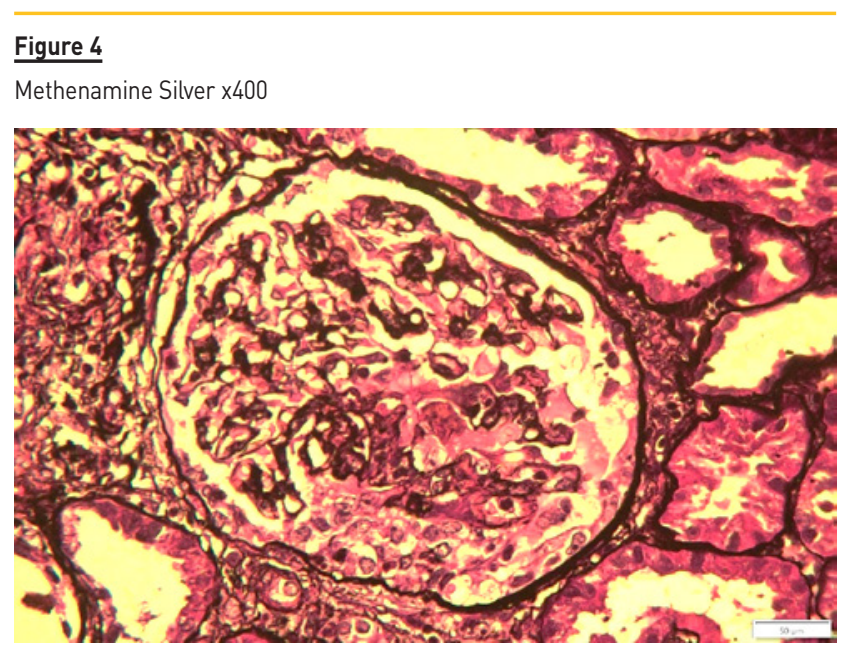

\section{QUESTIONS}

1. Given the above, what is the most likely diagnosis?

2. Considering the kidney biopsy specimen, what is the definitive diagnosis?

3. What is the most appropriate treatment in this situation? And prognosis?

\section{ANSWERS}

\section{Given the above, what is the most likely diagnosis?}

The most likely diagnosis is rapidly progressive GN, more precisely, ANCA-associated vasculitis (AAV). It is the most common form of newonset $\mathrm{GN}$ in adults over 50 years old and more frequent in male and caucasian patients ${ }^{1}$. The diagnosis relies on the combination of clinical findings and the results of imaging studies and basic and nonspecific biological tests (inflammatory markers such as erythrocyte sedimentation rate and C-reactive protein level, complete blood count, renal parameters, and urine sediment analysis). Our patient presented with constitutional symptoms, elevated inflammatory markers, fever and acute kidney dysfunction. Additionally, urine sediment revealed glomerular hematuria with dysmorphic erythrocytes and renal tubular cells, and he had a positive ANCA-PR3 titer of 9092UQ. Even with such overwhelming evidence, which prompted us to initiate treatment immediately, the definitive diagnosis is given solely by biopsy of the affected organ, when feasible. Renal biopsy is easier in comparison to biopsies of other organs and can show the classical pauci-immune crescentic and necrotizing GN in patients with renal involvement - and it was indeed our next step.

\section{Considering the kidney biopsy specimen, what is the definitive diagnosis?}

Although almost all pathological diagnoses made from a native kidney biopsy come from a careful examination of the renal cortex, certain diseases have a typical medullary component. Glomerulonephritis can

\section{Figure 5}

Periodic acid-Schiff $\times 400$

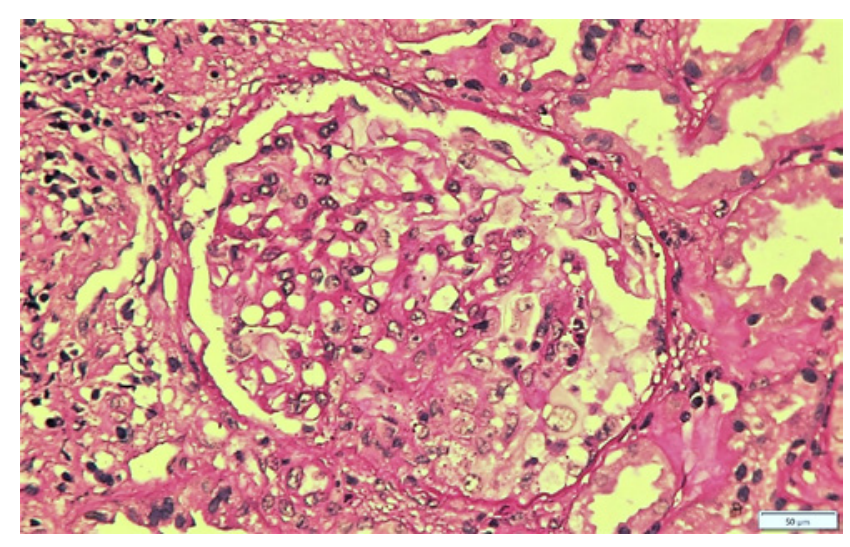

have characteristic cortical findings, namely in the glomeruli. However, certain disease entities remain elusive without close inspection of the medulla.

A kidney biopsy disclosed 10 glomeruli. The initial slides revealed intense interstitial inflammation with polymorphonuclears (Figure 1 and Figure 2) and one of seventeen glomeruli had a cellular crescent and fibrinoid necrosis (Figure 4 and Figure 5). The medulla had extensive interstitial hemorrhage and leukocytoclasia of neutrophils (Figure 3). Immunofluorescence performed in paraffin-embedded tissue was negative. As such, the diagnosis of pauci-immune glomerulonephritis with medullary angiitis was made.

Medullary angiitis is most commonly associated with AAV. Increased awareness of this pathologic finding is needed, as medullary angiitis can be ignored or misdiagnosed as acute interstitial nephritis or as an atypical finding in IgA nephropathy, and could be the only hint of severe systemic disease ${ }^{2}$.

3. What is the most appropriate treatment in this situation? And prognosis?

One of the first descriptions of medullary angiitis was by Watanabe et $a l$ in $1983^{4}$. Medullary angiitis is an uncommon finding in renal biopsies with rare descriptions in the literature and both its exact pathogenesis and best treatment remain unknown ${ }^{2}$. The paucity of studies describing this lesion is likely due to the fact that the focus of the examination of medical renal biopsies is on the findings in the renal cortex and medullary tissue is often not present for examination $^{3}$. These findings are thought to be due to thrombosis of the vasa recta with subsequent medullary hemorrhage. Reduced renal perfusion with relative medullary hypoxia has also been proposed for medulla-specific peritubular capillary involvement ${ }^{2}$. It is an important lesion to recognize as it commonly suggests the presence of systemic vasculitis and could be mistaken for interstitial nephritis. Differentiating between them is essential, considering the very contrasting etiologies, treatment, and prognosis. 
The induction regimen included intravenous methylprednisolone ( $1 \mathrm{~g} /$ day) for three consecutive days, followed by oral prednisolone (60mg/day) and intravenous cyclophosphamide $(750 \mathrm{mg})$. In spite of the highly immunosuppressive regimen that was chosen, Scr deteriorated further, and urgent dialysis was needed. As a last resort and regardless of the scarce evidence of benefit, we decided to start plasmapheresis. After the second dose of cyclophosphamide, five sessions of plasmapheresis and just one hemodialysis treatment, there was partial recovery of renal function, with serum creatinine of $4.2 \mathrm{mg} /$ $\mathrm{dL}$ at the time of hospital discharge. The patient has now completed 5 of the 6 planned doses of cyclophosphamide and is on prednisone taper, without substantial renal function recovery $(\mathrm{Scr} 4.01 \mathrm{mg} / \mathrm{dL}$, eGFR $14 \mathrm{~mL} / \mathrm{min} . / 1.73$ and protein/creatinine ratio of RPC $529.2 \mathrm{mg} / \mathrm{g}$ ). Even though there are no studies regarding the importance of the presence of medullary angiitis in the overall prognosis of patients with AAV, we can expect that the disease's course and treatment are similar to patients without it. However, this patient had an active glomerular inflammation far prior to the diagnosis establishment, and even though intensive immunosuppressive therapy was started, recovery of renal function was only partial. As such, this patient has a poor renal prognosis, and is expected to need renal replacement therapy in the medium term.

\section{References}

1. Pagnoux C. Updates in ANCA-associated vasculitis. European journal of rheumatology. 2016 Sep;3(3):122.

2. Klein J, Rodriguez $\mathrm{W}$, Kuperman $\mathrm{M}$, Szerlip $\mathrm{H}$. Medullary angitis and pauci-immune crescentic glomerulonephritis. InBaylor University Medical Center Proceedings 2017 Jul 1 (Vol. 30, No. 3, pp. 351-352). Taylor \& Francis.

3. Hendricks AR, Harris AA, Walker PD, Larsen CP. Renal medullary angiitis: a case series from a single institution. Human pathology. 2013 Apr 1;44(4):521-5.

4. Watanabe T, Nagafuchi Y, Yoshikawa Y, Toyoshima H. Renal papillary necrosis associated with Wegener's granulomatosis. Human pathology. 1983 Jun 1;14(6):551-7.

\section{Correspondence to:}

Filipa Cardoso, MD

Nephrology Department, Hospital de Curry Cabral - Centro Hospitalar de Lisboa Central

E-mail: cardoso.fsantos@gmail.com 